\title{
Multiscale Surveillance of Riemannian Manifolds
}

\author{
Henry Jacobs, Sujit Nair, and Jerrold Marsden \\ Control and Dynamical Systems \\ California Institute of Technology \\ \{hoj201, nair, marsden\}@cds.caltech.edu
}

\begin{abstract}
When surveying a surface, such as a spherical shell around a planet or a mine field, it is often desirable to survey a pre-chosen distribution while simultaneously respecting all its length scales. We review the Mix Norm developed by Mathew and Mezic [4] and their application for a multiscale coverage algorithm on the unit square and torus. We then develop tools to do multiscale surveillance on arbitrary Riemannian manifolds $(M, g)$. Next, we study the effects of applying the mix norm algorithm to more realistic scenarios where we have vehicles with different maximum speeds and frequency responses. We demonstrate this by applying the algorithm on the unit square to the case of a slow vehicle and a fast vehicle simultaneously exploring different spatial scales; we then test how well the uniform coverage algorithm allows a vehicle to estimate a function in a least squares sense. We also study the effects of varying the parameter $s$ in the definition of mix norm for $(M, g)$. In future work, we plan to apply a motions primitive based approach and explore ways of working with more general surfaces using triangular meshes.
\end{abstract}

\section{INTRODUCTION}

In many problems of scanning and surveillance, one often desires a trajectory to uniformly cover an entire space across various length scales. An initial attempt is to scan the area in the way that one typically mows a lawn. The drawbacks of this lawnmower approach are three-fold. Firstly, all effort is focused at a single length scale that is dependent upon the spacing between each row that is mowed. In terms of mine detection, if the mines are distributed proportional to $\sin (2 \pi x)$ on the $(x, y)$ plane, the lawn mower algorithm may erroneously report zero mines. Such an outcome results from implementing the lawn mower algorithm along the null set of $\sin (2 \pi x)$. Secondly, creating lawn mowing or milling trajectories for arbitrary domains is generally an NP-hard problem [1]. Thirdly, this particular method is not robust. If the lawnmower is kicked off course and displaced from the originally planned trajectory, some areas of the lawn will be doubly covered while other areas will be completely missed. One means of getting around such dilemmas is to choose a control law that generates a "mixing," as opposed to a merely ergodic (e.g. lawnmower-like), trajectory [2]. A trajectory that "mixes" can be viewed in terms of a dual problem of exploring all $L^{2}$ functions. Such a mixing flow will advect any scalar field into a uniform distribution, while ergodic but non-mixing vector fields will not. A byproduct of such a mixing strategy is that the search trajectories appear "chaotic". This has applications in randomized search strategies for police patrolling and airport security systems [3]. The flow of a constant non-commensurate vector field on $\mathbb{T}^{n}$ is the standard example of an ergodic but non- mixing trajectory. This flow suffers from the same problems as mentioned for the lawn mover algorithm. The formal definition of mixing is as follows

DEFINITION: $A$ flow $\phi_{t}$ on a measure space $(M, \mu)$ is mixing if for any two measurable sets $A$ and $B$, we have

$$
\lim _{t \rightarrow \infty}\left(\mu\left(A \cap \phi_{-t} B\right)\right)=\mu(A) \cdot \mu(B)
$$

From the standpoint of a probability theorist, this implies that the flow $\phi_{t}$ will send the correlation between "events" $A$ and $B$ to zero in infinite time. Thus, any finite perturbation of initial conditions will become statistically insignificant in infinite time (hence robustness), and no length scale should dominate another since such a property is independent of the size of $A$ and $B$ (hence multiscale).

Mathew et. al. have developed the "mix-norm" on $\mathbb{T}^{n}$ to measure how well a flow $\phi_{t}: \mathbb{T}^{n} \rightarrow \mathbb{T}^{n}$ mixes a scalar field $f \in L^{2}\left(\mathbb{T}^{n}\right)$ and proved that a trajectory is mixing on $\mathbb{T}^{n}$ if and only if the mix norm of the difference of the advected scalar field and it's average goes to zero (that is $\left\|\phi_{t}^{*} f-\bar{f}\right\|_{m i x} \rightarrow 0$ where $\phi^{*} f$ is the pull back of $f$ ) [4]. Recently, they devised a multi-scale coverage algorithm using a control law that points opposite the gradient of the mix norm, hence generating a rapidly mixing flow [5]. In this study, we begin to expand the mix norm to arbitrary Reimannian manifolds and apply the analogous multiscale coverage algorithm on $\mathbb{S}^{2}$. We show that such mix norms can also be used to tackle resource allocation problem in certain situations. For example, suppose we have two vehicles one of which is slower but with a very accurate sensor and the other being much faster but with a much cruder sensor. How do we optimally allocate tasks to them? One way to do this would be to send the fast but cruder sensor vehicle to make a large scale spatial survey of the region and send its feedback to the slower but accurate sensor for more fine tuned exploration. This task assignment can now be recast in the language of mix norm by assigning spatial scales (or frequency bands in Fourier domains) to each of these vehicles. We observe the emergent phenomena of the fast vehicle passing over the region of interest in quick sweeps, mostly paying attention to low frequency spatial modes, while the slower vehicle stays in the region handling higher spatial modes.

\section{A. Organization}

In section $\S$ II we begin the first steps of generalizing the mix norm to a larger class of Riemannian manifolds and use it to conjecture a plausible multiscale coverage algorithm. 
In section $\S I I I$ we perform the algorithm on the unit square and investigate the scenario of a fast and a slow vehicle simultaneously exploring the same space. In section $\S I V$ we investigate the algorithm on $\mathbb{T}^{2}$ and study how the algorithm behaves when we explicitly assign disjoint sets of spatial frequency modes to each vehicle. We also observe emergent behavior upon varying the $s$ parameter when viewing the mix norm as an $H^{s}$ norm. In section $\S \mathrm{V}$ we use the conjectures given at the end of section $\S$ II to create a multiscale coverage algorithm for $\mathbb{S}^{2}$. Finally we use a trajectory that uniformly coverages the unit square to estimate a function on that region in a least squares sense. Finally in section $\S \mathrm{VI}$ we discuss future directions to improve the work we've already done, and investigate questions that have arisen.

\section{The Mix Norm On Riemannian ManifoldS}

We implement the first steps in generalizing the mix norm on $\mathbb{T}^{n}$ introduced in [4] to a finite dimensional Riemannian manifold with finite global injectivity radius. Let $(M, g)$ be a compact connected finite dimensional Riemannian manifold. For two points $q_{1}, q_{2} \in M$, the distance $d\left(q_{1}, q_{2}\right)$ is defined by the length of a geodesic between them. Explicitly,

$$
d(x, y)=\inf \left\{\int_{0}^{1}\left(g\left(\gamma^{\prime}(t), \gamma^{\prime}(t)\right)^{1 / 2} d t\right\}\right.
$$

where the inf is taken over all differentiable curves $\gamma(t)$ with $\gamma(0)=q_{1}$ and $\gamma(1)=q_{2}$. The function $d: M \times M \rightarrow \mathbb{R}$ makes $(M, d)$ into a metric space. Using the induced measure $\mu=\sqrt{|g|} d q_{1} \wedge \cdots \wedge d q_{n}$ we can take a square integrable real valued function $f \in L^{2}(M)$ and define the linear operator $D(r): L^{2}(M) \rightarrow L^{2}(M)$

$$
(D(r) \cdot f)(q):=\frac{1}{\operatorname{vol}(B(q, r))} \int_{B(q, r)} f \mu,
$$

which produces the average of $f$ on a ball, $B(q, r)$, of radius $r$ centered at $q$. The function $D(r) \cdot f$ can be demonstrated to be square integrable. If the global injectivity radius is 1 then we can take the $L^{2}$ norm of this over all length scales and points of $M$ to get the mix norm

$$
\|f\|_{m i x}:=\left(\int_{0}^{1}\left(\int(D(r) \cdot f)(q) d q\right)^{2} d r\right)^{1 / 2} .
$$

In particular, if $\phi_{t}$ is a flow on $M$, then the pullback $\phi_{t}^{*} f=$ $f \circ \phi_{t}$ illustrates how the scalar field $f$ is advected by the flow, and $\left\|\phi_{t}^{*} f-\bar{f}\right\|_{M}$ measures how much the flow is "mixed," where $\bar{f}$ denotes the average of $f$ over all of $M$. It has been proven for $\mathbb{T}^{n}$ that the weak convergence of $\left\|\phi_{t}^{*} f-\bar{f}\right\|_{\text {mix }} \rightarrow$ 0 over a class of functions is equivalent to $\phi_{t}$ being mixing. The proof in [4] is as follow: we can define the mix norm as an inner product norm by using the the inner product of $f, g \in L^{2}(M)$

$$
\langle g, f\rangle=\int_{M} g(q) f(q) d q
$$

and the self adjoint operator $A$ on $L^{2}(M)$

$$
A:=\int_{0}^{1} D(r)^{*} D(r) d r
$$

From these definitions it follows

$$
\begin{aligned}
\|f\|_{\text {mix }}^{2} & =\int_{0}^{1}\langle D(r) \cdot f, D(r) \cdot f\rangle d r \\
& =\int_{0}^{1}\left\langle f, D(r)^{*} D(r) \cdot f\right\rangle d r \\
& =\langle f, A \cdot f\rangle
\end{aligned}
$$

Notice that $A$ is positive definite and self adjoint, and so if $\langle f, A \cdot f\rangle=0$ then $f=0$. In particular weak convergence of $\left\langle\phi_{t}^{*} f, \cdot\right\rangle$ to $\langle\bar{f}, \cdot\rangle$ is equivalent to convergence of $\| \phi_{t}^{*} f-$ $\bar{f} \|_{\text {mix }}$ to zero [4]. In [4] Mézic et. al. obtained a countable basis of $L^{2}\left(\mathbb{T}^{2}\right)$ from the eigenfunctions $e_{1}, e_{2}, \ldots$ of $\Delta_{g}$. There the $k^{t h}$ eigenvalue $\lambda_{k}$ is $|k|^{2}$, and they found that $e_{k}$ is also an eigenfunction of $A$ on $\mathbb{T}^{2}$ with an eigenvalue that decays like $\frac{1}{1+|k|^{2}}$. This induced an estimate of the mix norm on $\mathbb{T}^{2}$ by an equivalent Sobelev norm

$$
\|f\|_{\text {mix }}^{2} \approx \sum_{k_{1}, \ldots, k_{n}=0}^{\infty} \frac{1}{1+\sum_{i} k_{i}^{2}}\left\langle f, e_{k}\right\rangle^{2}
$$

Motivated by this we build the analogous estimate of the mix norm on $(M, g)$ as follows. For any Riemannian manifold $(M, g)$ the Laplace-Beltrami operator $\Delta_{g}$ written in coordinates as[6]

$$
\Delta_{g} f=(1 / \sqrt{g}) \sum_{j, k} \partial_{j}\left(g^{j k} \sqrt{g} \partial_{k} f\right)
$$

maps $C^{k}$ functions to $C^{k-2}$. It turns out that $\Delta_{g}$ has a countable set of eigenvalues with $C^{\infty}$ eigenfunctions forming a basis for $L^{2}(M)$. Using such a basis set of $L^{2}(M)$ induced by $\Delta_{g}$ denoted $e_{1}, e_{2}, \ldots$ with eigenvalues $\lambda_{1}, \lambda_{2}, \ldots$ we define the Sobelev norm for $f \in L^{2}(M)$ as

$$
\|f\|_{H^{s}}^{2}:=\sum_{k}^{\infty}\left(1+\lambda_{k}\right)^{-s}\left\langle f, e_{k}\right\rangle^{2} .
$$

We conjecture that the eigenvalues of the operator $A$ on $(M, g)$ decay like $\frac{1}{1+\lambda_{k}}$ enabling us to prove that the mix norm for $(M, g)$ as defined in 3 is equivalent to $\|f\|_{H^{s}}$ when $s=1$. We plan to study this question further as part of our future work. For example, on $\mathbb{S}^{2}$ with the standard inner product, an orthonormal basis of eigenfunctions of $\Delta_{g}$ in spherical coordinates is provided by the spherical harmonics $Y_{l}^{(m)}(\phi, \theta)$ with eigenvalues $l(l+1)$. We get that for $f \in L^{2}\left(\mathbb{S}^{2}\right)$

$$
\|f\|_{H^{s}}^{2}=\sum_{l=0,|m| \leq l}^{\infty}(1+l(l+1))^{-s}\left\langle f, Y_{l}^{(m)}\right\rangle^{2}
$$

so that the mixing norm is estimated when $s=1$. 


\section{Coverage on the Unit SQUARE}

In this section we study the effect of doing surveillance on a unit square using the mix norm when we have two vehicles with different forcing limits. The first part of this section follows the treatment in [5] closely. To construct the mix norm, one first starts with a basis function set for $L^{2}$ on the domain. The unit square being a manifold with boundary, one also needs to enforce some kind of boundary condition for the equation $\Delta_{g} f=\lambda f$. We choose to enforce the Dirichlet boundary conditions on the unit square. This provides basis functions

$$
f_{\mathbf{k}}=2 \cos \left(\pi k_{1} x\right) \cos \left(\pi k_{2} y\right)
$$

where $\mathbf{k}$ is the vector $\left(k_{1} k_{2}\right) \in \mathbb{Z} \times \mathbb{Z}$. These basis functions have eigenvalue $\pi^{2}\left(k_{1}^{2}+k_{2}^{2}\right)$ for $\Delta_{g}=\partial_{x}^{2}+\partial_{y}^{2}$, and so the mix norm of $f(x, y)$ is estimated with

$$
\|f\|_{m i x}^{2} \approx \sum_{\mathbf{k}} \Lambda_{\mathbf{k}}\left(\left\langle f, f_{\mathbf{k}}\right\rangle\right)^{2},
$$

where $\Lambda_{\mathbf{k}}=\left(1+\pi^{2}\left(k_{1}^{2}+k_{2}^{2}\right)\right)^{-1}$. For a trajectory $(x(t), y(t))$, we define the density $W_{t}$ by

$$
\left\langle W_{t}, f\right\rangle=\frac{1}{t} \int_{0}^{t} f(x(\tau), y(\tau)) d \tau .
$$

In order for $W_{t}$ to approach $\mu$ in the mix norm, we wish to minimize

$$
\Phi:=\left\|W_{t}-\mu\right\|_{m i x}^{2} \approx \sum_{\mathbf{k}} \Lambda_{\mathbf{k}}\left(\left\langle W_{t}-\mu, f_{\mathbf{k}}\right\rangle\right)^{2}
$$

as described in [5]. This leads to the control law

$$
\left(\begin{array}{c}
\dot{x} \\
\dot{y}
\end{array}\right)=-v_{\max } \frac{\vec{B}}{\|\vec{B}\|_{2}}
$$

where $\vec{B}=\sum_{\mathbf{k}} \Lambda_{\mathbf{k}}\left(\left\langle W_{t}-\mu, f_{\mathbf{k}}\right\rangle \nabla f_{\mathbf{k}}\right.$. In the case of a double integrator where the velocity can only be controlled indirectly by its second derivative, one gets a similar control law:

$$
\left(\begin{array}{l}
\ddot{x} \\
\ddot{y}
\end{array}\right)=-F_{\max } \frac{\vec{B}}{\|\vec{B}\|_{2}} .
$$

We use this control law with a damping term in the next section.

\section{A. Vehicles with different forcing terms}

In the case of vehicles with different forcing terms, each may end up dealing with a different set of length scales as an emergent phenomena from obeying the given control law. We consider a slower vehicle with much damping and a vehicle that exerts greater forces with little damping. We will refer to the faster vehicle as the "airplane" and the slower vehicle as the "car" out of convenience and not from any implementation of realistic plane or car modeling in this case. Often the vehicles may leave the unit square, in which case they are pushed back with maximum forcing. We observe that the airplane maneuvers huge sweeps away from the center, while the car slowly covers what the plane
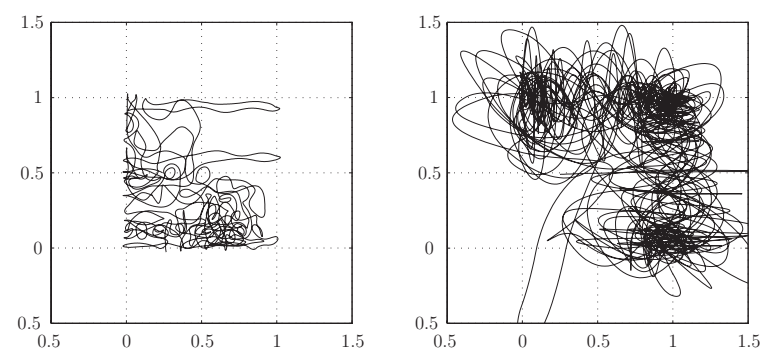

Fig. 1: Trajectories for two vehicles moving simultaneously on the same unit square according to control law (11) with different max forcing coefficients. We've displayed the trajectories on separate plots for ease of viewing Left: trajectory of the slow vehicle with $F_{\max }=0.1$ and damping coefficient 1.0. Right: trajectory of fast vehicle with $F_{\max }=1.0$ and damping coefficient 0.1 .

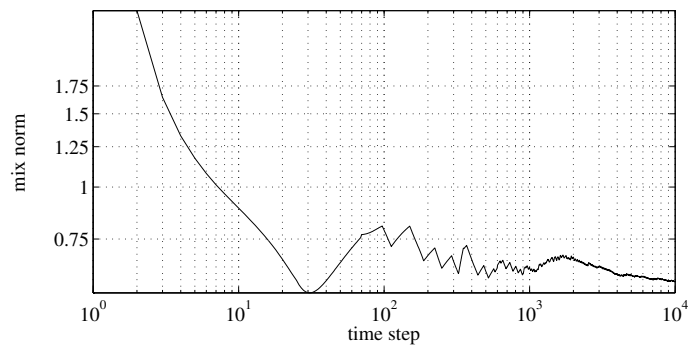

Fig. 2: Evolution of the mix norm for vehicles with different maximum forcing.

missed (see trajectories in figure (1) and the mix norm in figure (2)). Thus we see an emergent phenomena where the controller automatically assigns different spatial scales to the vehicles depending upon their forcing limits. The faster airplane explores large spatial scales (low frequencies) and often passes the boundary where as the slower car explores small spatial scales (high frequencies) in more detail and remains confined to the interior of the unit square.

\section{B. Function Estimation}

Given a scalar function $f \in L^{2}(M)$ we create an algorithm for a vehicle on $M$ that will estimate $f$ with only knowledge of it's trajectory up to time $t$ and the value of $f$ along it (that is the set of points $\{(x(\tau), f(x(\tau))): \tau \leq t\}$ ). Such a method is similar to using a flashlight on a large painting in a dark room in order to guess the picture. In particular, if the flashlight passes across every region, one will be able to make a well-informed guess of what the picture is. We implement this idea as follows: We generate a discretized trajectory $\left\{x_{i}: i=1,2, \ldots, n\right\}$ from the control law in equation (10) that covers the region uniformly. We then choose the Fourier coefficient $\hat{f}_{k}$ of $f$ on the some orthonormal basis, $e_{k}(x)$, to minimize: 

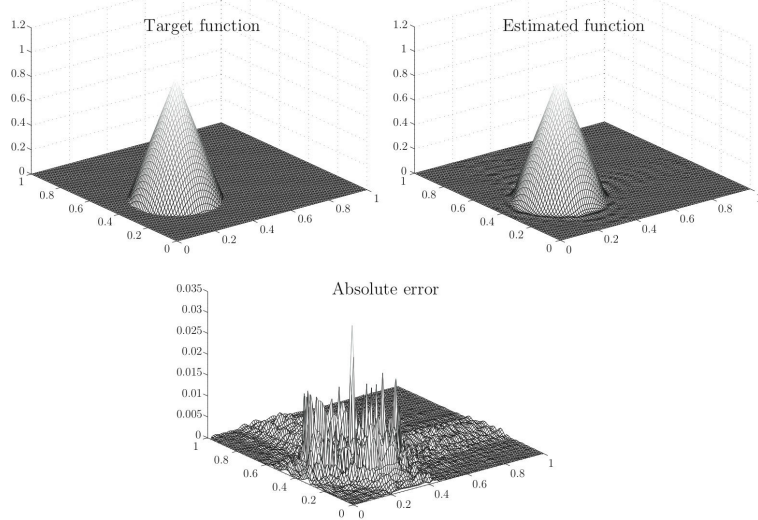

Fig. 3: Top left: the target function, $f$, defined in equation (12). Top right: The estimated function, $f$, from 6000 sample points after integrating 6000 time steps. Bottom: Absolute error $\mid f(x, y)-$ $\tilde{f}(x, y) \mid$.

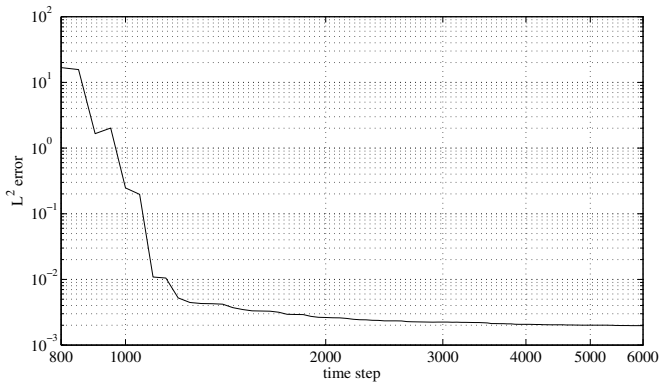

Fig. 4: The evolution of $L^{2}$ error for the estimation of function $f$ defined in (12) using trajectories generated by mix nom controller (10).

$$
E\left(\left\{f_{k}\right\}\right)=\sqrt{\sum_{i k}\left(\hat{f}_{k} e_{k}\left(x_{i}\right)-f\left(x_{i}\right)\right)^{2}}
$$

This is a finite dimensional least squares problem with solution

$$
\hat{f}_{k}=\left[G G^{T}\right]^{-1}[G] \cdot f\left(x_{i}\right)
$$

Where $G:=\left[G_{j}^{i}\right]=\left[e_{i}\left(x_{j}\right)\right]$. Using the basis given in 9 we test this on the unit square for a uniform coverage algorithm with a single integrator with maximum speed 1 , and timestep $h=0.05$ for Fourier modes $k \leq 30$. The target function we chose is

$$
f(x, y)=\left\{\begin{array}{l}
1-5 r \text { if } r<0.2 \\
0 \text { else }
\end{array}\right.
$$

where $r(x, y)=\sqrt{(x-0.3)^{2}+(y-0.4)^{2}}$, this forms a cone centered at point $(0.3,0.4)$ with height 1 and radius 0.2 . The target function, it's estimate, and the absolute error are shown after 6000 time-steps in figure (3). It's worth noting that most of the error that can be handled with 31 modes along each coordinate is taken care of much sooner as shown in plot (4). In fact, 31 modes along each coordinate direction makes for 961 basis functions, and we observe a rapid decay in the error centered around that time, which is the time where the number of data points we've gathered begins to exceed the number Fourier coefficients we wish to calculate. Most of the error that can be handled with this many modes is eaten after 2000 time steps.

\section{Coverage ON $\mathbb{T}^{2}$}

In section $\S$ III-A, we saw an emergent phenomena where the controller automatically assigns spatial scales to the vehicles depending upon their forcing limits. In $\S I V-A$, we will show how this can be enforced even in the case of two vehicles with similar forcing or similar speed limits. The main idea is to explicitly assign to these vehicles different frequency bands over which the explore the domain. In $\S$ IV$\mathrm{B}$, we will explore what happens when one varies $s$ in the $H^{s}$ norm. Note that $s=1$ corresponds to the mix norm. One would expect that as one increases $s$, the trajectories would get smoother because more weight will be placed on the lower spatial modes. Conversely the acceptable set of distributions can be of a higher frequency nature. This is because, if a function lies in $H^{s}$, its Fourier components decays at least as fast as $\left|\lambda_{k}\right|^{s}$. Therefore, this additional parameter $s$ could be potentially used to tweak the smoothness level of vehicle trajectory depending upon its frequency response. For this section, the domain we consider is $\mathbb{T}^{2}$ with the standard inner products $g=d x \otimes d x+d y \otimes d y$. This induces the operator $\Delta_{g}=\partial_{x}^{2}+\partial_{y}^{2}$, which in turn induces the basis functions

$$
\left\{e_{\left(k_{0}, k_{1}\right)}\left(\theta_{0}, \theta_{1}\right)=l_{k_{0}}\left(\theta_{0}\right) \cdot l_{k_{1}}\left(\theta_{1}\right): k_{0}, k_{1} \in \mathbb{Z}\right\}
$$

where

$$
l_{k}(x)= \begin{cases}\cos (k x) & \text { for } k>0 \\ \frac{1}{2 \pi} & \text { for } k=0 \\ \sin (k x) & \text { for } k<0\end{cases}
$$

and the mix norm is

$$
\|f\|_{m i x}^{2} \approx \sum_{k_{0}, k_{1}=0}^{\infty} \frac{1}{1+k_{1}^{2}+k_{0}^{2}}\left\langle f, e_{k_{0}, k_{1}}\right\rangle^{2}
$$

\section{A. Mode Assignment}

We observe frequent tradeoffs between speed and both maneuverability (the ability to make sharp turns) and detection (i.e. sensor resolution). In either case, commanding a vehicle to maneuver at frequencies so high that can neither be achieved nor enable sensors to scan is a wasted effort. Using the control law (10), sharp turns will arise when adding in the higher Fourier modes to the velocity. One step to acknowledging this redundancy in more realistic systems is to restrict the Fourier modes involved in the control law of each vehicle. In particular, assigning a restricted set of low modes to the vehicle that can only implement low frequency responses, such as a faster vehicle with low resolution sensors. We use assign the remaining high frequencies to another vehicle that is capable of responding to such high frequency commands. For the vehicle that only addresses low frequency modes, we use the first 5 spatial modes along one 


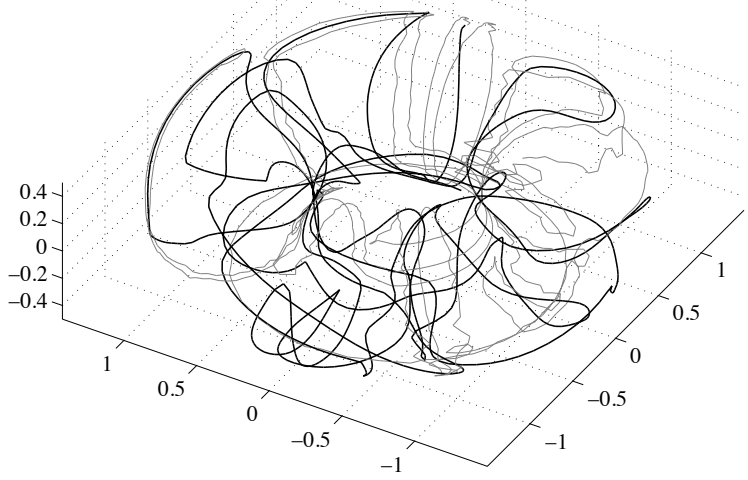

Fig. 5: Trajectories of the high mode (black) and low mode (grey) vehicles on the $\mathbb{T}^{2}$ with cut-off frequencies $k_{0}=5$ and $k_{1}=10$ with top speed $M=1.0$

angle of the torus, and the first 10 spatial modes along the other. This gives us the control law on the 2-torus:

$$
\begin{aligned}
\dot{x_{1}} & =-M \frac{\overrightarrow{B_{1}}}{\left\|\overrightarrow{B_{1}}\right\|} \\
\overrightarrow{B_{1}} & =\sum_{k_{0}<5, k_{1}<10} \Lambda_{k_{0}, k_{1}}\left\langle W_{t}-\mu, e_{k_{0}, k_{1}}\right\rangle \nabla e_{k_{0}, k_{1}}\left(x_{1}(t)\right)
\end{aligned}
$$

and for the high-mode vehicle, we account for the higher modes with the control law

$$
\begin{aligned}
\dot{x}_{2} & =-M \frac{\vec{B}_{2}}{\left\|\vec{B}_{2}\right\|} \\
\vec{B}_{2} & =\sum_{k_{0} \geq 5, k_{1} \geq 10} \Lambda_{k_{0}, k_{1}}\left\langle W_{t}-\mu, e_{k_{0}, k_{1}}\right\rangle \nabla e_{k}\left(x_{2}(t)\right) .
\end{aligned}
$$

where $\Lambda_{k_{0}, k_{1}}=\left(1+k_{0}^{2}+k_{1}^{2}\right)^{-1}$. The results are shown in figure (5). We see that the vehicle is assigned the lower frequency band $\left(k_{0}<5, k_{1}<10\right)$ explores large spatial scales (black) where as the vehicle which is assigned higer frequency band ( $\left.k_{0} \geq 5, k_{1} \geq 10\right)$ explores small spatial scales (grey).

\section{$B$. Varying $s$ in the the $H^{s}$ norm on the 2-torus}

The $H^{s}$ Sobolev norm in Fourier space is

$$
\|f\|_{H^{s}}^{2}=\sum_{k}\left(1+\|k\|^{2}\right)^{-s}\left\langle f, e_{k}\right\rangle^{2} .
$$

with $s=1$ corresponding to the mix norm. Written as above, the the parameter $s$ provides a new degree of freedom and any $s \geq 0$ will provide a different Sobelev norm. Intuitively, larger $s$ will make the resulting norm less sensitive to highly oscillatory modes. We show trajectories for $s=0,1,2$ in figure (6) and we see that they get smoother for increasing values of $s$.

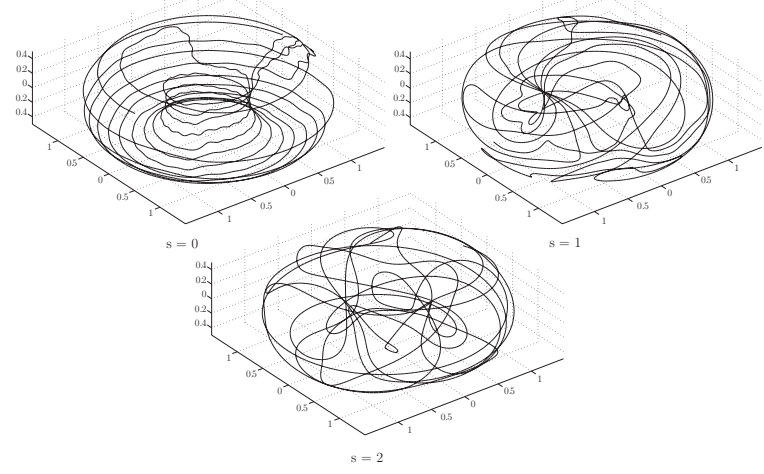

Fig. 6: Trajectories with mix norm replaced by Sobelev norm with $s=0,1,2$

\section{Coverage ON $\mathbb{S}^{2}$}

As a final example of coverage on a Riemannian manifold, we consider the case of a two dimensional sphere $\mathbb{S}^{2}$ with standard inner product $g=\sin (\theta) d \phi \otimes d \phi+d \theta \otimes d \theta$ in spherical coordinates with azimuthal angle $\theta$ and longitudinal angle $\phi$. One can think of the problem of planet exploration using autonomous vehicles or of a satelling trying to map out a planet. For the sphere case, the mixing norm or $H^{1}$ norm is defined in terms of spherical harmonics $e_{l m}=Y_{l}^{(m)}(\theta, \varphi)$, which form a set of smooth basis function for $L^{2}\left(\mathbb{S}^{2}\right)$. We approximate the mixing norm on $\mathbb{S}^{2}$ with the spherical harmonics $l=0, \ldots, 20$ by defining the density

$$
\Phi(c)^{2}=\sum_{l=0,|m| \leq l}^{20} \frac{1}{1+l(l+1)}\left\langle c, e_{l m}\right\rangle
$$

We define the time average density $W_{t}$ corresponding to a trajectory $c(t)$ by

$$
\left\langle W_{t}, f\right\rangle=\frac{1}{t} \int_{0}^{t} f(c(t)) d t
$$

In order for $W_{t}$ to approach density $\mu$, we set $c^{\prime}(t)$ such that we maximize the instantaneous decrease in $\Phi^{2}$. We then

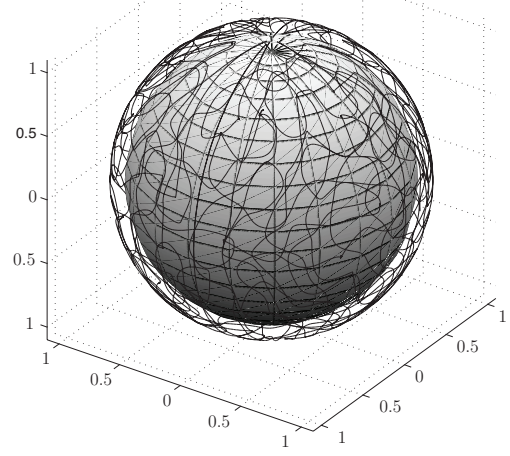

Fig. 7: A trajectory approaching a uniform distribution $\mu=\frac{1}{4 \pi}$ on $\mathbb{S}^{2}$ with $l_{\max }=20$ 


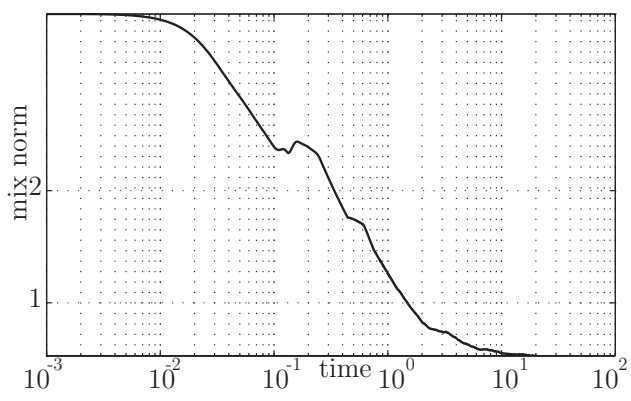

Fig. 8: Decay of the mix norm $\left\|W_{t}-\frac{1}{4 \pi}\right\|_{\mathbb{S}^{2}}$

obtain the control law:

$$
c^{\prime}(t)=-v_{\max } \frac{\vec{B}}{\|\vec{B}\|_{2}}
$$

where

$$
\vec{B}=\sum \frac{1}{1+l(l+1)}\left(\left\langle W_{t}-\mu, e_{l m}\right\rangle \nabla e_{l m}\right.
$$

Figure (7) shows a simulated trajectory for coverage of a sphere with the decay of the mix norm as shown in Figure (8).

\section{FUTURE WORK}

We have yet to prove that the mix-norm defined in equation (3) is actually equivalent to the Sobelev norm defined in equation (8). A key component of reaching such a relation involves the use of a mean value property for eigenfunctions of $\Delta_{g}$. In $\mathbb{R}^{n}$ and $\mathbb{T}^{n}$ the mean value operator, $D(s)$, shares eigenfunctions with $\Delta_{g}$ and the eigenvalues of $D(s)$ decay like $(1+\lambda)^{-1}$ for each eigenvalue $\lambda \in \sigma\left(\Delta_{g}\right)$. We are currently combing the literature for sufficient conditions. None the less, the use of the Sobelev norm in 8 from the eigenfunctions of $\Delta_{g}$ is enough to ensure that $W_{t}$ weakly converges to a distribution $\mu$ in the $H^{s}$ topology since such eigenfunctions will form a complete basis for $L^{2}(M)$.

In the specific case of dynamics on $\mathbb{S}^{2}$, we are currently concerned with the coordinate singularity that comes with using spherical coordinates. One option is to lay an FEMtype grid on the sphere embedded in $\mathbb{R}^{3}$, and calculate the eigenfunctions of the the graph Laplacian on this grid. If such a method succeeds, we seek to implement it on a large class of compact manifolds including non-smooth surfaces such as polytopes.

In regards to function estimation, we are currently investigating links to compressed sensing, and robustness to sensor noise. We suspect improvements to our algorithm can be improved by minimizing the $L^{1}$ error rather than the $L^{2}$ error as suggested by compressed sensing literature [7], where function estimation is found by evaluating the function on randomly placed lines.

One commonality in search algorithms is the time evolution of the desired distribution as new data is learned. A naive implementation for changing the distribution requires re-computing all Fourier coefficients. However, this need not be the case if the nature of the distribution evolution is known. In the example of land mine detection, the probability distribution may continually adapt to account for new information, such as metal detected at location $x$. The programmer might respond to such new findings by adding a normal distribution centered at $x$ to the current distribution, and re-normalizing. Likewise, rather than re-computing all of the Fourier coefficients, it may be easier to pre-compute those of a normal distribution, taking translations into account, and add these coefficients to the old ones and normalize them afterwards.

Additionally we plan to implement a motion primitives based algorithm for minimizing the mix norm in order to make problems involving realistic dynamics more tractable. In the case of helicopter dynamics, for example, the nonholonomic constraints can be difficult to consider in rigorously deriving a control law. One way to get around such complications is to pre-compute optimized trajectories offline to move from one point to another. During simulation, the pre-computed trajectories can be executed sequentially (subject to boundary conditions). Choosing the motion primitive that minimizes the mix norm at each time step would be a possible control law in this instance.

Finally, the control laws (10) and (11) both presume perfect communication between vehicles so that only the most recently updated density $W_{t}$ is used. In many scenarios however, such as helicopter dynamics, communication is limited to local exchange. As part of using the motion primitives to make this algorithm more feasible, it would be wise to account for the inconveniences of imperfect and short range communication.

\section{ACKNOWLEDGMENTS}

This research was supported by a Graduate Research Fellowship from the Rose Hills Foundation. We owe special thanks to Phillip du Toit and Marin Kobilarov for general comments and suggestions.

\section{REFERENCES}

[1] E. M. Arkin, S. P. Fekete, and J. S. B. Mitchell, Approximation Algorithms for Lawn Mowing and Milling, Computational Geometry, vol 17, pp 25-50, 2000

[2] A. Lasota, M. Mackey, An Introduction to Fractals and Chaos, Second Edition, Applied Mathematical Sciences, vol 97, Springer-Verlag, 1994

[3] P. Paruchuri, M. Tambe, F. Ordonez, and S. Kraus. Security in multiagent systems by policy randomization. In AAMAS '06: Proceedings of the fifth international joint conference on Autonomous agents and multiagent systems, pages 273-280, New York, NY, USA, 2006. ACM.

[4] G. Mathew, I. Mezic, L. Petzold, A Multiscale Measure for Mixing, Physica D: Nonlinear Phenomena, vol.211, pp. 23-46, November 2005

[5] G. Mathew, I. Mezic, Spectral Multiscale Coverage: A Uniform Coverage Algorithm for Mobile Sensor Networks, Proceedings of the 48th IEEE Conference on Decisions and Control, 2009.

[6] I. Chavel, Eigenvalues in Riemannian Geometry, Academic Press, 1984

[7] E. J. Candes, J. K. Romberg Signal Recovery from Random Projections In Computational Imaging III. Edited by Bouman, Charles A.; Miller, Eric L. Proceedings of the SPIE, Volume 5674, pp. 76-86, 2005 